\title{
A LABORATORY EXPERIMENT ON THE NUCLEATION OF INTERSTELLAR DUSTS
}

\author{
F. KA MIJO, Y. NAKA DA, T. IGUCHI, M. FUJIMOTO, \\ M. TAKADA, and S. TAKA JO \\ Dept. of Astronomy, University of Tokyo, Japan
}

\begin{abstract}
Small particles of silica, carbon, and iron are produced in the laboratory for the eventual purpose of studying their optical properties.
\end{abstract}

Very fine powders of some silicates, carbon and iron are produced by the gas evaporation technique in argon gas. After evacuating the working chamber of an evaporation unit, argon is introduced to a given pressure. A tungsten heater in the chamber is then gradually heated to evaporate the material. The evaporated atoms are cooled by collision with those of the argon gas and when the temperature of the vapour becomes lower than its dew point, fine particles are formed in the atmosphere of the argon gas.

To investigate the size and shape of the particles, several standard grid meshes coated with carbon film for electron microscopy are put in the chamber. Generally the size becomes smaller for lower temperature and also for lower pressure. The largest size

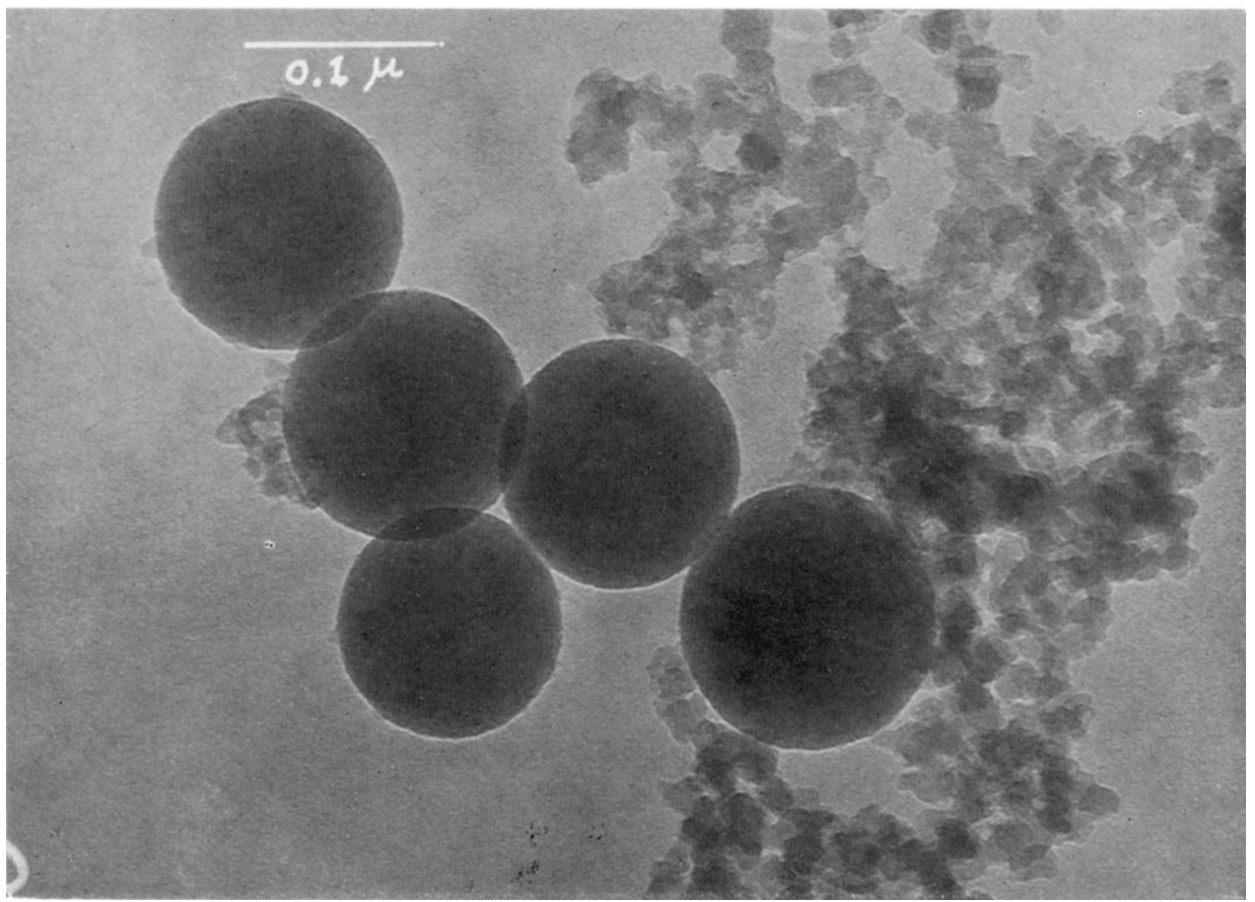

Fig. 1. $\mathrm{SiO}_{2}$. 


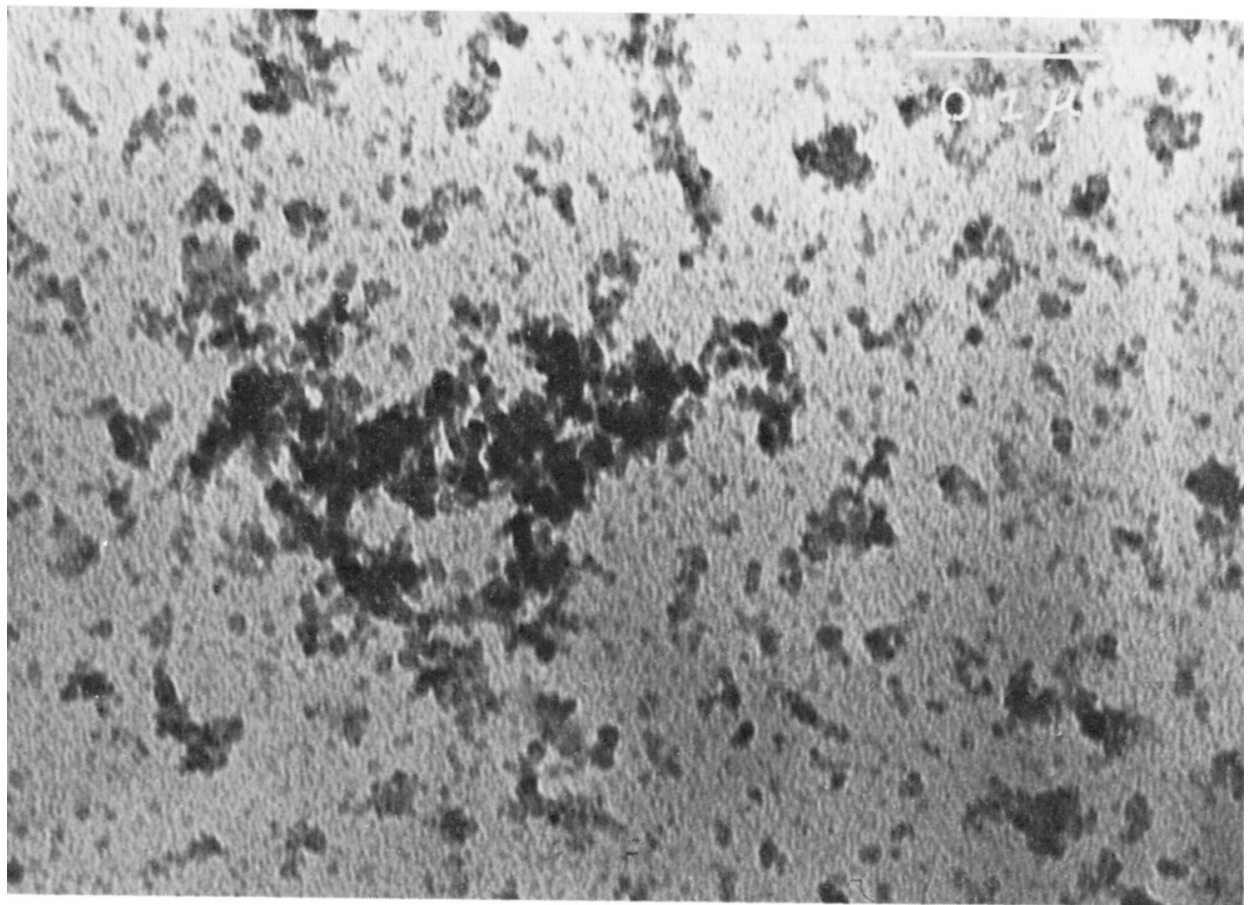

Fig. 2. C.

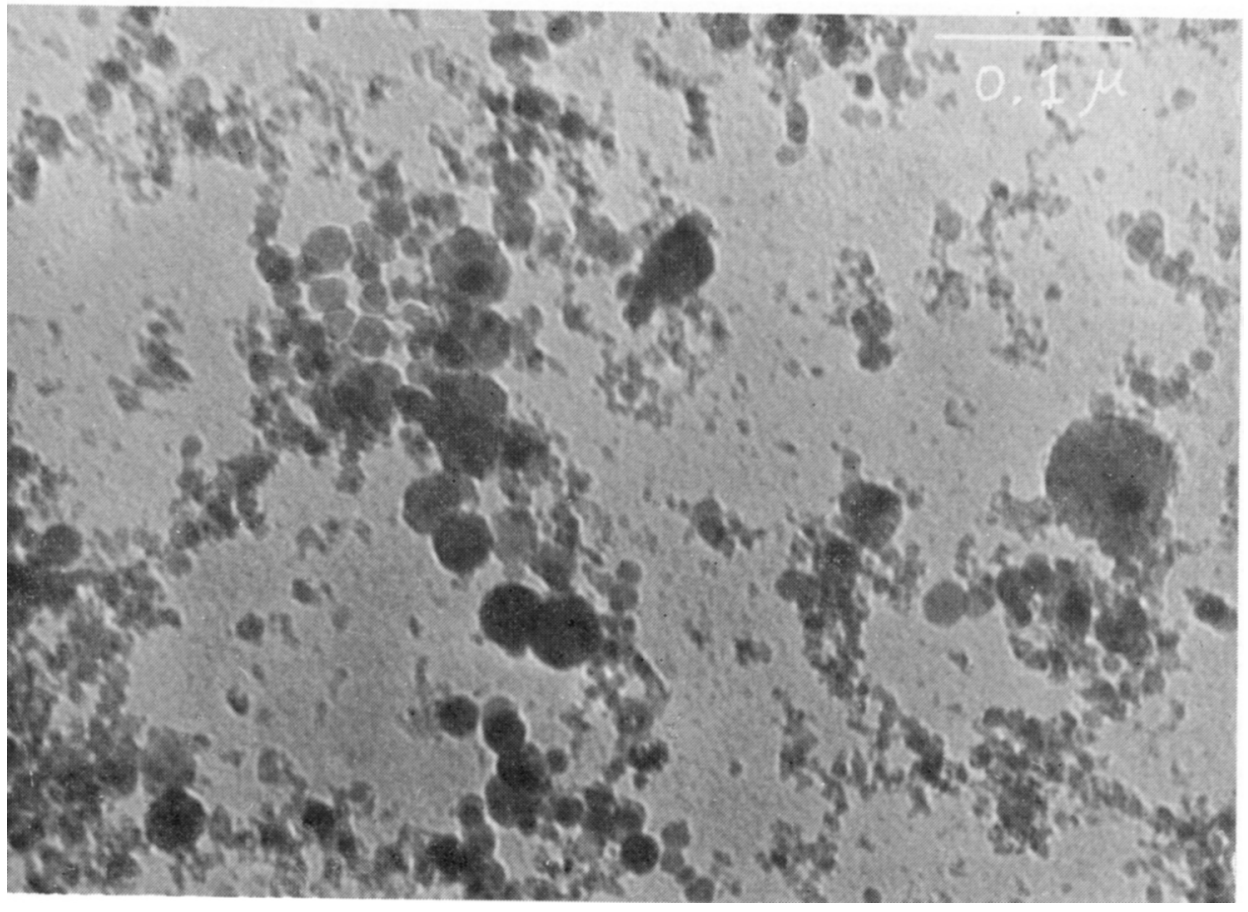

Fig. 3. Fe. 
we have obtained is about $0.1 \mu$ for silica and the smallest one is about $40 \AA$ for carbon. (see Figures 1 and 2).

\section{Silica (Figure 1)}

Instead of the evaporation unit, an ordinary plasma jet is used to produce silica particles. A broken piece of silica glass is placed on the water cooled copper hearth. Then the working chamber of the system is filled with argon gas of one atmosphere. While the plasma jet gun is fired, the hearth is operated carefully so that the flame shoots the specimen. Silica glass melts inmmediately. Fine particles are produced in the argon atmosphere and deposit on the inside walls of the chamber. As we expect from their spherical shape, they show no sign of crystal structure by electron diffraction.

\section{Carbon (Figure 2)}

Fine particles of carbon are obtained by striking the arc in argon gas of 9 torr. They also show no crystal structure.

\section{Iron (Figure 3)}

Iron particles prepared by the arc in argon gas of 6 torr show characteristic hexagonal shape. It is said that they are rhombic dodecahedron.

A detailed report on the experiment including the UV and IR absorption of the particles will be published in Publ. Astron. Soc. Japan. 